\title{
A Supersymmetric Black Ring
}

\author{
Henriette Elvang, ${ }^{1, *}$ Roberto Emparan, ${ }^{2, \dagger}$ David Mateos, ${ }^{3, \dagger}$ and Harvey S. Reall ${ }^{4,8}$ \\ ${ }^{1}$ Department of Physics, University of California, Santa Barbara, California 93106-9530, USA \\ ${ }^{2}$ ICREA, Departament de Física Fonamental, and C.E.R. en Astrofísica, Física de Partícules i Cosmologia, \\ Universitat de Barcelona, Diagonal 647, E-08028 Barcelona, Spain \\ ${ }^{3}$ Perimeter Institute for Theoretical Physics, Waterloo, Ontario, Canada N2J 2 W9 \\ ${ }^{4}$ Kavli Institute for Theoretical Physics, University of California, Santa Barbara, California 93106-4030, USA
}

(Received 14 July 2004; published 18 November 2004)

\begin{abstract}
A new supersymmetric black hole solution of five-dimensional supergravity is presented. It has an event horizon of topology $S^{1} \times S^{2}$. This is the first example of a supersymmetric, asymptotically flat black hole of nonspherical topology. The solution is uniquely specified by its electric charge and two independent angular momenta. These conserved charges can be arbitrarily close, but not exactly equal, to those of a supersymmetric black hole of spherical topology.
\end{abstract}

DOI: 10.1103/PhysRevLett.93.211302

A major success of string theory is the statisticalmechanical explanation of the Bekenstein-Hawking entropy of certain supersymmetric black holes. The original example is the five-dimensional black hole studied in [1]. This is also the simplest example, as it carries the minimum number of net charges necessary to have a finitearea regular horizon, namely, D1-brane and D5-brane charges and linear momentum along an internal direction. A generalized solution with the same charges and equal angular momenta in two orthogonal planes was discovered, and its entropy microscopically reproduced, by Breckenridge, Myers, Peet, and Vafa (BMPV) [2], thus extending the success of [1] to rotating black holes with a single independent rotation parameter.

The BMPV black hole has a topologically spherical event horizon. It has recently been realized that this is not true of all five-dimensional rotating black holes: the vacuum Einstein equations admit a (nonsupersymmetric) black ring solution, with horizon topology $S^{1} \times S^{2}$ [3]. The existence of black rings raises the question of whether there are any supersymmetric black holes in five dimensions other than BMPV.

In [4] it was proven that the geometry of the event horizon of any supersymmetric black hole of minimal five-dimensional supergravity must be (i) $T^{3}$, (ii) $S^{1} \times S^{2}$, or (iii) (possibly a quotient of) a homogeneously squashed $S^{3}$. It was also proven that the only asymptotically flat supersymmetric solution with horizon geometry (iii) is the BMPV black hole (which reduces to a solution of minimal supergravity when its three charges are set equal). The purpose of this Letter is to present a solution of type (ii), that is, a supersymmetric black ring. Such a solution was conjectured to exist in [5] motivated by the work of [6].

This is the first example of an asymptotically flat supersymmetric solution with a regular event horizon of nonspherical topology. It possesses a richer structure than the BMPV solution, which we see arises as a particular case. It is parametrized by its electric charge and two indepen-
PACS numbers: 04.70.Dy, 04.65.+e, 11.10.Wx, 11.30.Pb

dent angular momenta, which illustrates the fact that supersymmetry imposes no constraint on the angular momenta. It also has a nonvanishing magnetic dipole, which is fixed by the asymptotic charges and therefore is not an independent parameter. Some black rings are believed to be unstable [3] but supersymmetry should ensure that this new solution is stable.

Our solution corresponds to taking equal values for the three charges (D1, D5, and momentum) and three dipoles (D1, D5, and Kaluza-Klein monopole) of a more general supersymmetric black ring (or, viewed in higher dimensions, a black supertube [7] with three charges [5]). The details of these will be given elsewhere [8], but we do anticipate that, although the equal-charge solution presented here is entirely determined by its conserved charges, this is not the case for those of [8].

Progress in understanding how the string microscopic description of black holes distinguishes between different horizon topologies has recently been made [9]. The existence of the supersymmetric black ring opens for the first time the exciting possibility of studying this question for a black hole with a regular horizon of finite area in a supersymmetric, highly controlled, setting. We leave this and other questions raised by the existence of our solution for the future.

The solution.-The bosonic sector of five-dimensional minimal supergravity is Einstein-Maxwell theory with a Chern-Simons term. Any supersymmetric solution of this theory must possess a nonspacelike Killing vector field $V$ [10]. In a region where $V$ is timelike, the metric can be written as [11]

$$
d s^{2}=-f^{2}(d t+\omega)^{2}+f^{-1} d s^{2}\left(M_{4}\right)
$$

where $V=\partial / \partial t, M_{4}$ is an arbitrary hyper-Kähler space, and $f$ and $\omega$ are a scalar and a one-form on $M_{4}$, respectively, which must satisfy [11]

$$
d G^{+}=0, \quad \Delta f^{-1}=\frac{4}{9}\left(G^{+}\right)^{2},
$$


where $G^{+} \equiv \frac{1}{2} f(d \omega+\star d \omega)$, with $\star$ the Hodge dual on $M_{4}$ and $\Delta$ is the Laplacian on $M_{4}$.

For our solution, $M_{4}$ is just flat space $\mathbb{R}^{4}$, whose metric we write as $[3,9]$

$$
\begin{aligned}
d s^{2}\left(\mathbb{R}^{4}\right)= & \frac{R^{2}}{(x-y)^{2}}\left[\frac{d y^{2}}{y^{2}-1}+\left(y^{2}-1\right) d \psi^{2}+\frac{d x^{2}}{1-x^{2}}\right. \\
& \left.+\left(1-x^{2}\right) d \phi^{2}\right] .
\end{aligned}
$$

The coordinates have ranges $-1 \leq x \leq 1$ and $-\infty<y \leq$ -1 , and $\phi, \psi$ have period $2 \pi$. Asymptotic infinity lies at $x \rightarrow y \rightarrow-1$. Note that the apparent singularities at $y=$ -1 and $x= \pm 1$ are merely coordinate singularities, and that $(x, \phi)$ parametrize (topologically) a 2 -sphere. The locus $y=-\infty$ is, in (3), a circle of radius $R>0$ parametrized by $\psi$. In the full geometry (1) it will be blown up into a ring-shaped horizon. The orientation is $\epsilon_{y \psi x \phi} \equiv 1$.

The scalar and one-form of the solution are given by

$$
f^{-1}=1+\frac{Q-q^{2}}{2 R^{2}}(x-y)-\frac{q^{2}}{4 R^{2}}\left(x^{2}-y^{2}\right)
$$

and $\omega=\omega_{\psi}(x, y) d \psi+\omega_{\phi}(x, y) d \phi$, with

$$
\begin{aligned}
& \omega_{\phi}=-\frac{q}{8 R^{2}}\left(1-x^{2}\right)\left[3 Q-q^{2}(3+x+y)\right], \\
& \omega_{\psi}=\frac{3}{2} q(1+y)+\frac{q}{8 R^{2}}\left(1-y^{2}\right)\left[3 Q-q^{2}(3+x+y)\right] .
\end{aligned}
$$

$Q$ and $q$ are positive constants, proportional to the net charge and to the local dipole charge of the ring, respectively. We assume $Q \geq q^{2}$, so that $f^{-1} \geq 0$. Note that $\omega$ is smooth at finite $y$ since $\omega_{\phi}(x= \pm 1)=\omega_{\psi}(y=-1)=0$ (i.e., there are no Dirac-Misner string pathologies). In verifying that (4) and (5) solve (2) it is useful to observe that $\left(1-x^{2}\right) \omega_{\psi, x}=\left(y^{2}-1\right) \omega_{\phi, y}$. The Maxwell field strength $F=d A$ is uniquely determined by $f$ and $\omega$ [11]. For our solution the gauge potential is

$$
A=\frac{\sqrt{3}}{2}\left[f(d t+\omega)-\frac{q}{2}[(1+x) d \phi+(1+y) d \psi]\right] .
$$

The metric (3) can be brought to a manifestly flat form with the coordinate transformation

$$
\rho \sin \Theta=\frac{R \sqrt{y^{2}-1}}{x-y}, \quad \rho \cos \Theta=\frac{R \sqrt{1-x^{2}}}{x-y} .
$$

In these coordinates the solution takes the form

$$
f^{-1}=1+\frac{Q-q^{2}}{\Sigma}+\frac{q^{2} \rho^{2}}{\Sigma^{2}}
$$

$$
\begin{aligned}
\omega_{\phi}= & -\frac{q \rho^{2} \cos ^{2} \Theta}{2 \Sigma^{2}}\left[3 Q-q^{2}\left(3-\frac{2 \rho^{2}}{\Sigma}\right)\right], \\
\omega_{\psi}= & -\frac{6 q R^{2} \rho^{2} \sin ^{2} \Theta}{\Sigma\left(\rho^{2}+R^{2}+\Sigma\right)} \\
& -\frac{q \rho^{2} \sin ^{2} \Theta}{2 \Sigma^{2}}\left[3 Q-q^{2}\left(3-\frac{2 \rho^{2}}{\Sigma}\right)\right],
\end{aligned}
$$

where $\Sigma \equiv \sqrt{\left(\rho^{2}-R^{2}\right)^{2}+4 R^{2} \rho^{2} \cos ^{2} \Theta}$. Using these coordinates it is straightforward to see that if we set $R=0$ then the solution reduces to the BMPV black hole.

Symmetries and charges. - The results of [11] imply that our black ring preserves four supersymmetries. It has isometry group $\mathbb{R} \times \mathrm{U}(1)^{2}$, whereas BMPV has $\mathbb{R} \times$ $\mathrm{U}(1) \times \mathrm{SU}(2)$. The mass and angular momenta of the solution follow from its manifestly asymptotically flat form (8) and (9) as

$$
\begin{gathered}
M=\frac{3 \pi}{4 G} Q, \quad J_{\phi}=\frac{\pi}{8 G} q\left(3 Q-q^{2}\right), \\
J_{\psi}=\frac{\pi}{8 G} q\left(6 R^{2}+3 Q-q^{2}\right) .
\end{gathered}
$$

The total electric charge $\mathbf{Q}$ is proportional to $Q$ and satisfies $M=(\sqrt{3} / 2) \mathbf{Q}$; hence the Bogomol'nyi-PrasadSommerfeld (BPS) inequality of [10] is saturated.

Absence of closed timelike curves (CTCs).-As $y \rightarrow$ $-\infty$ we find

$$
g_{\psi \psi}=3\left[\frac{\left(Q-q^{2}\right)^{2}}{4 q^{2}}-R^{2}\right]+\frac{q^{2}}{4}\left(1-x^{2}\right)+\mathcal{O}\left(y^{-1}\right),
$$

so we demand

$$
R<\frac{Q-q^{2}}{2 q}
$$

to ensure that $\partial / \partial \psi$ remains spacelike. This condition is sufficient to avoid any CTCs at finite $y$. To see this, consider the function $(x-y)^{2} f^{-2} g_{\psi \psi} /(-1-y)$, which is a polynomial in $x$ and $y$. This can be grouped into a sum of terms that are all non-negative if (11) holds. In the same manner, one can check that the determinant of the $2 \times 2$ metric $g_{i j}, i, j=\phi, \psi$ is always non-negative. These two conditions are necessary and sufficient for $g_{i j}$ to be positive semidefinite.

The event horizon.-To examine what happens as $y \rightarrow$ $-\infty$ it is convenient to define a new coordinate $r=$ $-R / y$. Now consider a coordinate transformation of the form

$$
\begin{gathered}
d t=d v-B(r) d r, \quad d \phi=d \phi^{\prime}-C(r) d r, \\
d \psi=d \psi^{\prime}-C(r) d r,
\end{gathered}
$$

where

$$
B(r)=\frac{B_{2}}{r^{2}}+\frac{B_{1}}{r}+B_{0}, \quad C(r)=\frac{C_{1}}{r}+C_{0} .
$$

The electromagnetic potential is regular in the new coordinates up to terms that can be removed by a gauge 
transformation. The constants $B_{i}$ and $C_{i}$ are chosen so that all metric components remain finite as $r \rightarrow 0$. To eliminate a $1 / r$ divergence in $g_{r \psi^{\prime}}$ and a $1 / r^{2}$ divergence in $g_{r r}$ we choose $B_{2}=q^{2} L /(4 R)$ and $C_{1}=-q /(2 L)$, where

$$
L \equiv \sqrt{3\left[\frac{\left(Q-q^{2}\right)^{2}}{4 q^{2}}-R^{2}\right]},
$$

which is positive as a consequence of (11). To avoid a $1 / r$ divergence in $g_{r r}$ we need $B_{1}=\left(Q+2 q^{2}\right) /(4 L)+$ $L\left(Q-q^{2}\right) /\left(3 R^{2}\right)$. The metric is then analytic at $r=0$ with $g_{r r}$ a linear function of $x$ at $r=0$. We can eliminate this function by choosing the finite part of the coordinate transformation as follows: $C_{0}=-\left(Q-q^{2}\right)^{3} /\left(8 q^{3} R L^{3}\right)$, $B_{0}=q^{2} L /\left(8 R^{3}\right)+2 L /(3 R)-R /(2 L)+3 R^{3} /\left(2 L^{3}\right)+$ $3\left(Q-q^{2}\right)^{3} /\left(16 q^{2} R L^{3}\right)$. The metric can now be written

$$
\begin{aligned}
d s^{2}= & -\frac{16 r^{4}}{q^{4}} d v^{2}+\frac{2 R}{L} d v d r+\frac{4 r^{3} \sin ^{2} \theta}{R q} d v d \phi^{\prime} \\
& +\frac{4 R r}{q} d v d \psi^{\prime}+\frac{3 q r \sin ^{2} \theta}{L} d r d \phi^{\prime}+2\left[\frac{q L}{2 R} \cos \theta\right. \\
& \left.+\frac{3 q R}{2 L}+\frac{\left(Q-q^{2}\right)\left(3 R^{2}-2 L^{2}\right)}{3 q R L}\right] d r d \psi^{\prime}+L^{2} d \psi^{\prime 2} \\
& +\frac{q^{2}}{4}\left[d \theta^{2}+\sin ^{2} \theta\left(d \phi^{\prime}-d \psi^{\prime}\right)^{2}\right]+\ldots
\end{aligned}
$$

where $x=\cos \theta$ and the ellipsis denotes terms involving subleading (integer) powers of $r$ in all of the metric components explicitly indicated, as well as terms in $g_{r r}$ starting at $\mathcal{O}(r)$. (We have not displayed the leading order term in $g_{r r}$ because it is lengthy and unilluminating.) The determinant of this metric is analytic in $r$. At $r=0$ it vanishes if, and only if, $\sin ^{2} \theta=1$, which is just a coordinate singularity. It follows that the inverse metric is also analytic in $r$ and hence the above coordinates define an analytic extension of our solution through the surface $r=0$.

The supersymmetric Killing vector field $V=\partial / \partial v$ is null at $r=0$. Furthermore, $V_{\mu} d x^{\mu}=(R / L) d r$ at $r=0$, so $V$ is normal to the surface $r=0$. Hence $r=0$ is a null hypersurface and a Killing horizon of $V$; i.e., the black ring has an event horizon at $r=0$.

If $L=0$, then a similar analysis shows that the geometry has a null orbifold singularity instead of an event horizon.

Horizon geometry.-We can read off the geometry of a spatial cross section of the event horizon:

$$
d s_{\text {horizon }}^{2}=L^{2} d \psi^{\prime 2}+\frac{q^{2}}{4}\left(d \theta^{2}+\sin ^{2} \theta d \chi^{2}\right),
$$

where $\chi \equiv \phi^{\prime}-\psi^{\prime}=\phi-\psi$. We see that the horizon has geometry $S^{1} \times S^{2}$, where the $S^{1}$ and $S^{2}$ have radii $L$ and $q / 2$, respectively. Note that the $S^{2}$ is round, in contrast with nonextremal black rings, for which the $S^{2}$ is deformed in the $\theta$ direction.

The area of the event horizon is

$$
\mathcal{A}=2 \pi^{2} L q^{2}=\pi^{2} q \sqrt{3\left[\left(Q-q^{2}\right)^{2}-4 q^{2} R^{2}\right]} .
$$

The surface gravity and angular velocities of the event horizon vanish, as expected for a supersymmetric, asymptotically flat black hole [12]. The horizon is at infinite proper spatial distance from points outside it; i.e., it lies down an infinite throat.

Near-horizon limit.-The near-horizon limit is defined by $r=\epsilon L \tilde{r} / R, v=\tilde{v} / \epsilon$, and $\epsilon \rightarrow 0$. In this limit, the metric becomes

$$
\begin{aligned}
d s^{2}= & 2 d \tilde{v} d \tilde{r}+\frac{4 L}{q} \tilde{r} d \tilde{v} d \psi^{\prime}+L^{2} d \psi^{\prime 2}+\frac{q^{2}}{4}\left(d \theta^{2}\right. \\
& \left.+\sin ^{2} \theta d \chi^{2}\right) .
\end{aligned}
$$

This is the product of a spacetime that is locally $\mathrm{AdS}_{3}$ (anti-de Sitter) with radius $q$ and a 2 -sphere of radius $q / 2$, as expected from [4]. The $\mathrm{AdS}_{3}$ space is the nearhorizon geometry of an extremal Banados-TeitelboimZanelli black hole of horizon radius $r_{+}=L$. This nearhorizon limit is not the same as the "decoupling limit" relevant to the $\mathrm{AdS}_{3} / \mathrm{CFT}_{2}$ (conformal field theory) duality, which will be analyzed in [8].

Infinite-radius limit.-The existence of a supersymmetric black ring solution was recently conjectured in [5,13]. As evidence, Ref. [13] constructed a black string solution that was claimed to describe a black ring of infinite radius. This solution can indeed be recovered as the infinite-radius limit of our solution as follows. Define a charge density $\bar{Q}=Q / 2 R$ and new coordinates $r=$ $-R / y, \eta=R \psi$, and $\cos \theta=x$. In the limit $R \rightarrow \infty$ with $\bar{Q}, q, r$, and $\eta$ held fixed we have

$$
\begin{gathered}
f^{-1} \rightarrow 1+\frac{\bar{Q}}{r}+\frac{q^{2}}{4 r^{2}}, \\
\omega_{\psi} d \psi \rightarrow-\left(\frac{3 q}{2 r}+\frac{3 q \bar{Q}}{4 r^{2}}+\frac{q^{3}}{8 r^{3}}\right) d \eta,
\end{gathered}
$$

and $\omega_{\phi} \rightarrow 0$. This is the solution of [13] for the special case of three equal charges and three equal dipoles. In [8] we shall present a more general supersymmetric black ring whose infinite-radius limit is the general solution of [13]. Note that the solution of [13] does not exhibit an essential feature of the supersymmetric black ring, namely, the existence of two independent angular momenta.

Dipole charge.-Define

$$
\mathcal{D}=\frac{1}{16 \pi G} \int_{S^{2}} F=\frac{\sqrt{3}}{16 G} q,
$$

where the $S^{2}$ is a surface of constant $t, \psi$, and $y$ outside the horizon. This "charge" determines the radius of the $S^{2}$ of the horizon and also (for fixed electric charge) the angu- 


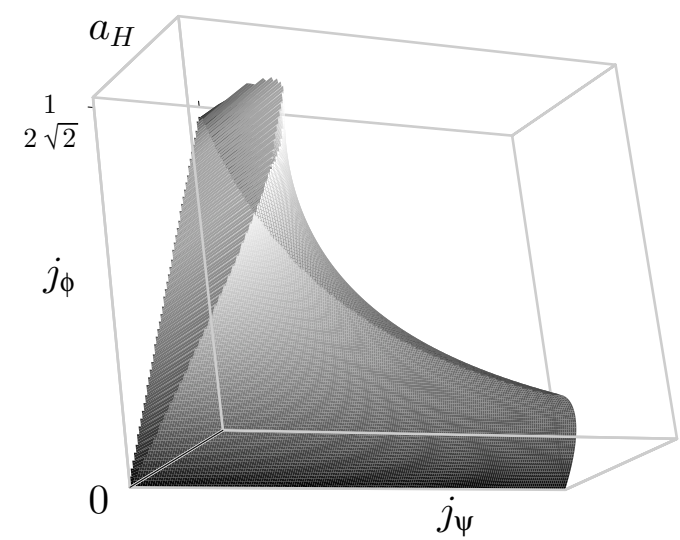

FIG. 1. Plot of the dimensionless horizon area $a_{H}=$ $\mathcal{A} /(G M)^{3 / 2}$ as a function of the dimensionless angular momenta $j_{i}=[27 \pi /(32 G)]^{1 / 2} J_{i} / M^{3 / 2}, i=\psi, \phi$. The scales for $j_{\phi}$ and $j_{\psi}$ are different for a better representation: the planar boundary corresponds to $j_{\psi}=j_{\phi}$ (which is reached only as $R \rightarrow 0$ ). The surface extends to infinity to the right.

lar momentum $J_{\phi}$. It is not conserved except in the limit in which the ring becomes an infinite black string. The general solution of [8] carries three independent dipole charges, which are proportional, respectively, to the number of D1-branes, D5-branes, and Kaluza-Klein (KK) monopoles with a world-volume direction around the ring circle. The solution presented here corresponds to taking equal values for these three dipole charges, so $q$ is proportional to the number of branes with a worldvolume direction around the ring circle. When oxidized to six dimensions, the black ring becomes a black supertube. We anticipate that regularity of this solution will lead to $q$ being quantized in units of the radius of the KK circle, since $q$ is the number of KK monopoles making up the tube [8].

Uniqueness.-The supersymmetric black ring is uniquely specified by its electric charge and angular momenta. Figure 1 shows the region of the $J_{\phi}-J_{\psi}$ plane occupied by BPS black rings for fixed charge $Q$. There are three boundaries to this region. The boundary to the upper right arises from the condition (11) with $L$, the radius of the $S^{1}$, vanishing at the boundary. The lower boundary $J_{\phi} \rightarrow 0$ arises from the condition $q>0$ so the radius of the $S^{2}$ vanishes at this boundary. The leftmost, straight boundary arises from the condition $R>0$, which implies $J_{\psi}>J_{\phi}$. If $R=0$ the solution reduces to the BMPV solution, so there are no black rings with $J_{\psi}=J_{\phi}$, and thus the conserved charges of our black ring are always different from those of a BMPV black hole.

Entropy.-Figure 1 displays the black ring horizon area as a function of the angular momenta for fixed charge $Q$. For fixed $J_{\phi}$ the entropy function is maximized as $R \rightarrow 0$. However, this function is not continuous at $R=0$. At this point, the black ring solution reduces to the BMPV black hole, whose entropy is greater than the $R \rightarrow 0$ limit of the black ring entropy. This discontinuity is due to the change in the horizon topology from $S^{1} \times S^{2}$ at $R>0$ to $S^{3}$ at $R=0$, and is analogous to the discontinuous increase in entropy that occurs when two sources of a multicenter extremal Reissner-Nordstrom solution become coincident.

The existence of supersymmetric black rings raises many questions. For example: Are there any supersymmetric black hole solutions (of minimal supergravity) for the empty regions of the $J_{\phi}-J_{\psi}$ plane, or any solutions that overlap with the currently covered regions? If not, then can the results of [4] be strengthened to a full uniqueness theorem for supersymmetric black holes? Is there a general nonextremal black ring solution that reproduces our solution and those of $[3,9]$ as special cases? Such a solution would presumably depend on five parameters corresponding to the angular momenta, the electric charge, the dipole charge, and the mass. Finally, is it possible to perform a statistical-mechanical calculation of the entropy of this black ring?

We thank V. Balasubramanian, B. Cabrera-Palmer, J. Gauntlett, D. Marolf, J. Simón, and especially G. Horowitz and R. Myers, for useful discussions. H. E. was supported by the Danish Research Agency and NSF Grant No. PHY-0070895. R. E. was supported in part by UPV00172.310-14497, FPA2001-3598, DURSI 2001SGR-00188, and HPRN-CT-2000-00131. H. S. R. was supported in part by the National Science Foundation under Grant No. PHY99-07949.

\footnotetext{
*Electronic address: elvang@physics.ucsb.edu

†Electronic address: emparan@ub.edu

${ }^{\ddagger}$ Electronic address: dmateos@perimeterinstitute.ca

${ }^{\S}$ Electronic address: reall@kitp.ucsb.edu
}

[1] A. Strominger and C. Vafa, Phys. Lett. B 379, 99 (1996).

[2] J. C. Breckenridge, R. C. Myers, A.W. Peet, and C. Vafa, Phys. Lett. B 391, 93 (1997).

[3] R. Emparan and H. S. Reall, Phys. Rev. Lett. 88, 101101 (2002).

[4] H. S. Reall, Phys. Rev. D 68, 024024 (2003); 70, 089902 (2004).

[5] I. Bena and P. Kraus, Phys. Rev. D 70, 046003 (2004).

[6] O. Lunin and S. D. Mathur, Phys. Rev. Lett. 88, 211303 (2002); S. D. Mathur, A. Saxena, and Y. K. Srivastava, Nucl. Phys. B680, 415 (2004).

[7] D. Mateos and P. K. Townsend, Phys. Rev. Lett. 87, 011602 (2001).

[8] H. Elvang, R. Emparan, D. Mateos, and H.S. Reall, hep-th/0408120.

[9] H. Elvang, Phys. Rev. D 68, 124016 (2003); H. Elvang and R. Emparan, J. High Energy Phys. 0311 (2003) 035; R. Emparan, J. High Energy Phys. 0403 (2004) 064.

[10] G.W. Gibbons et al., Nucl. Phys. B416, 850 (1994).

[11] J. P. Gauntlett et al., Classical Quantum Gravity 20, 4587 (2003). We follow the conventions of this paper except for the metric signature, which we take to be positive.

[12] J. P. Gauntlett, R. C. Myers, and P. K. Townsend, Classical Quantum Gravity 16, 1 (1999).

[13] I. Bena, hep-th/0404073. 